\title{
A sudden neurological deficit in a patient with endocarditis
}

\author{
Andrea Alberti · Giancarlo Agnelli · \\ Mohammed Hamam · Stefano Pasquino • \\ Piero Floridi · Temistocle Ragni · Maurizio Paciaroni
}

Received: 16 January 2009/ Accepted: 19 January 2009/Published online: 19 February 2009

(C) SIMI 2009

\section{Case record}

Dr. Alberti, Dr. Pasquino, Dr. Hamam, Prof. Agnelli: A 20-year-old woman was admitted to a community hospital for a continuous high-grade fever. She reported fatigue and weight loss over the previous month. The history was otherwise negative. A trans-thoracic echocardiography showed a vegetation on the mitral valve associated with mild regurgitation. Blood cultures were positive for Staphylococcus hominis and specific antibiotic therapy was initiated. Because of persistent sepsis and signs of heart failure, the patient was referred to the Cardiac Surgery Unit of our hospital, and surgery was performed. The procedure was done during extracorporeal circulation through a median sternotomy, and utilising cold cardioplegic cardiac arrest. Intra-operative findings included: rupture of the cordae tendineae and erosion of the valvular posterior leaflet of the mitral valve, papillary

\footnotetext{
A. Alberti $(\bowtie) \cdot$ G. Agnelli · M. Paciaroni

Stroke Unit, Department of Internal and Cardiovascular

Medicine, Santa Maria della Misericordia Hospital,

University of Perugia, S. Andrea delle Fratte,

06156 Perugia, Italy

e-mail: andrea_alberti@hotmail.com

M. Hamam · P. Floridi

Department of Neuroradiology,

Santa Maria della Misericordia Hospital,

Perugia, Italy

S. Pasquino $\cdot$ T. Ragni

Department of Cardiac Surgery,

Santa Maria della Misericordia Hospital,

Perugia, Italy
}

muscles and the posterior left ventricular wall. Additionally, fragile endocarditic vegetations were detected. After removal of the infected tissue, an autologous pericardial patch was used to restore the ventricular and valvular anatomy.

Three days after surgery, the patient presented with a sudden headache with right hemiparesis and aphasia, and was referred to our Stroke Unit. A cerebral computed tomography (CT scan) revealed a hemorrhagic lesion in the left frontal-parietal hemisphere (Fig. 1). Digital subtraction angiogram of the left internal carotid artery showed a ruptured large aneurysm $(1 \mathrm{~cm}$.) in the distal frontal branch of the left middle cerebral artery (Fig. 2). An intracranial mycotic (infectious) aneurysm (IAA) was diagnosed because of associated endocarditis and morphological features, so endovascular embolisation was performed. A microcatheter (Excel 14, Boston Scientific Corporation) was super-selectively advanced to the parent artery, and extended until it reached the mycotic aneurysm. Embolisation was performed using $N$-butyl-cyanoacrylate glue (Braun, Germany) with occlusion of the aneurysm and the parent artery (Fig. 3). Over the next few hours, the patient underwent craniotomy for the evacuation of intraparenchymal haematoma due to a deterioration of her clinical condition that was related to the mass effect of the haemorrhagic lesion.

Antibiotic therapy was continued, and an echocardiography examination showed no recurrence of endocarditis. Follow-up angiography performed 30 days later confirmed the complete exclusion of the aneurysm. Thus, no recurrences and no re-treatments were needed. No further cerebral complications occurred, and the patient was transferred to a rehabilitation centre 1 month later. One year later, aphasia and hemiparesis were improved with rehabilitation. 


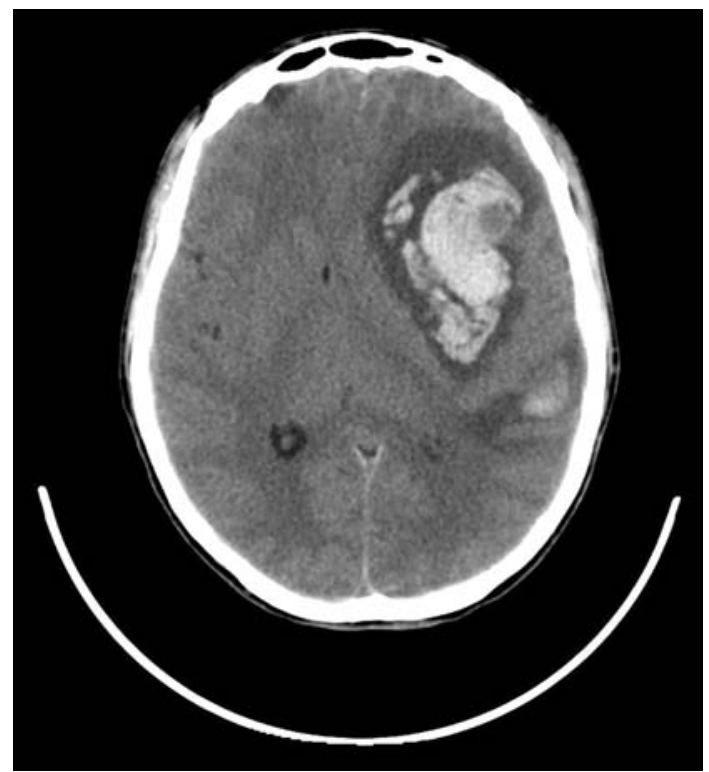

Fig. 1 CT scan of the brain shows large left tempero-frontal intracerebral haematoma

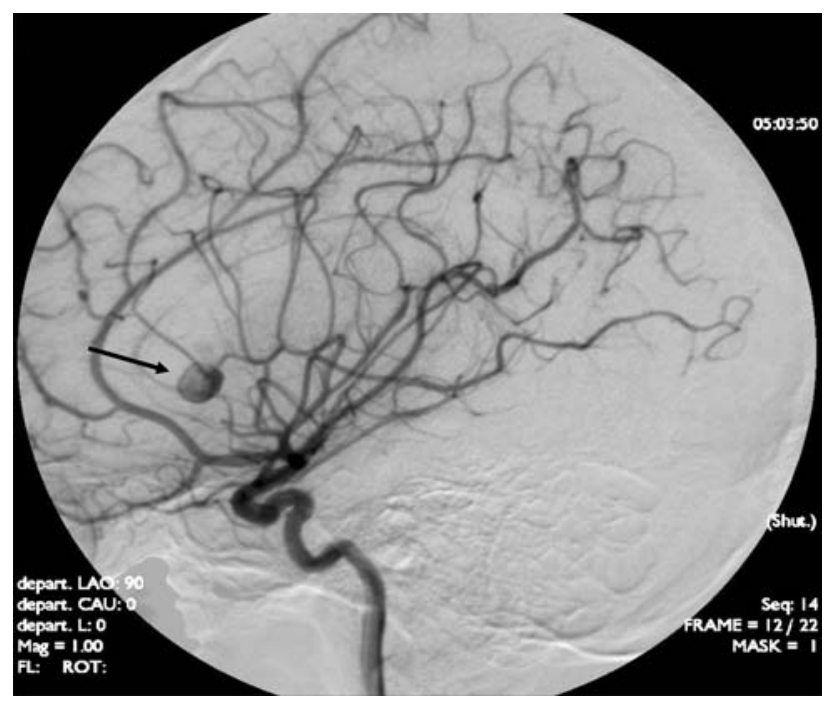

Fig. 2 Digital subtraction angiogram of the left internal carotid artery, shows a ruptured large mycotic aneurysm $(1 \mathrm{~cm}$.) in the distal frontal branch of middle cerebral artery

\section{Discussion of intracranial infectious aneurysms diagnosis}

Dr. Paciaroni, Dr. Floridi, Prof. Ragni, Dr. Alberti: Although cerebrovascular complications of bacterial endocarditis are common [1], the development of mycotic aneurysms related to endocarditis are rare [2]. Intracranial infectious aneurysms (IIAs), or so called mycotic aneurysms, account for $0.7-5.4 \%$ of all intracranial aneurysms [3] and almost all of these have been observed in patients with infective endocarditis [4].

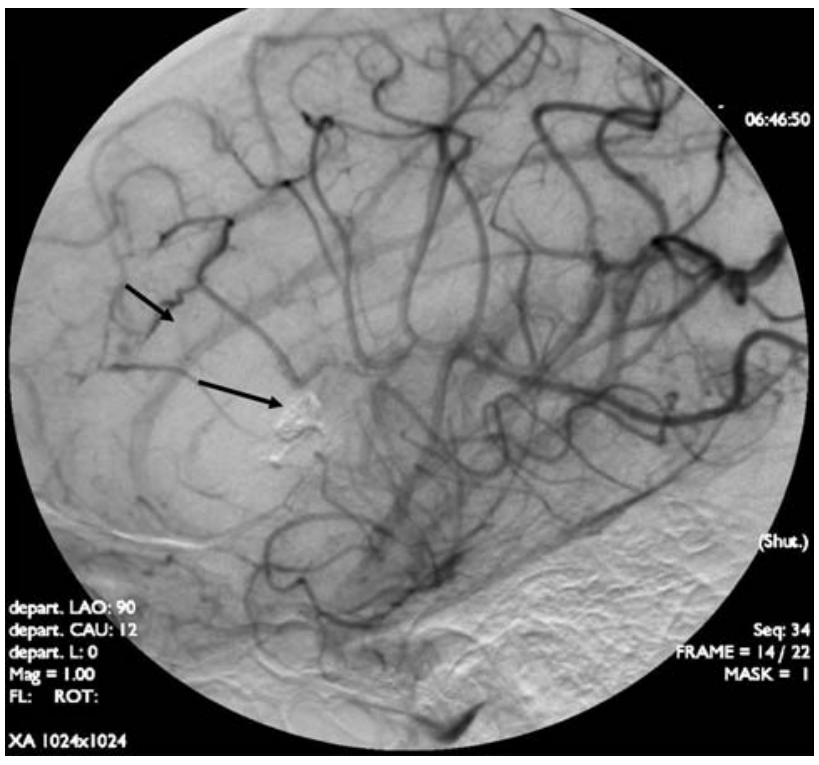

Fig. 3 Lateral view of the left internal carotid artery after occlusion of the parent artery and the sac of aneurysm with cyanoacrylate. The distal segment is patent and refilled by collateral vessels

Intracranial infectious aneurysm development is due to the circulation of infective emboli that reach the distal cerebral arteries and destroy the integrity of these vessels, with disruption of internal elastic membrane and intima [5]. IIA most often occur within the first 2-4 weeks of antimicrobial therapy [6]. Because septic embolisation is often clinically silent, the incidence of IIA is probably higher than the reported 2-4\% in endocarditis series [7]. Staphylococcus and Streptococcus species are the most causative pathogens involved [3,5]. IIAs are more common in the anterior cerebral circulation, and are more frequently located in the middle cerebral artery, have irregular morphology, are quite friable and lack the classical neck showed by saccular aneurysms [8]. Although the formation of IIAs is rare, they represent a potentially devastating neurological complication with high morbidity and mortality, especially among patients having ruptured IIAs [2].

Headache, confusion, or focal neurological deficits can indicate the presence of IIA development, but these are common neurological symptoms of patients with endocarditis [9]. Thus, unfortunately, aneurysms are often suspected only after their rupture. In the presence of a suspected IIA, cerebral CT scan is the best "first-test". Multidetector CT angiography and magnetic resonance angiography (MRA) imaging are minimally invasive techniques for the detection of IIAs, although the conventional four-vessel angiography remains the gold standard. 


\section{Discussion of intracranial infectious aneurysms treatment}

Dr. Hamam, Dr. Paciaroni, Dr. Alberti: The optimal management of IIAs is undefined, as no randomised controlled trials have been carried out to date. Current modalities including conservative antibiotic treatment, surgical therapy, and more recently, endovascular therapy (EVT) are available. Although the general condition of the patient, location of IIAs, features and number of aneurysms can influence the treatment, the most important factor is whether an aneurysm has ruptured or not.

A ruptured aneurysm is a neurological emergency, and an early exclusion of a ruptured IIA is highly imperative because of the high risk of re-rupturing. The surgical approach is the classic option [10], but has some difficulties. The risks of surgery are greater for patients with large or ruptured mycotic aneurysms, while distal aneurysms can be missed with a surgical approach. Furthermore, a direct clipping of aneurysms whose walls are acutely inflamed and friable is potentially more dangerous. Finally, not all patients are suitable candidates for surgery due to their complicated physical conditions $[5,9,10]$. Recent literature has reported several cases that describe the use of EVT as a safe alternative treatment for IIAs [4, 10, 11]. EVT seems to have several advantages over surgery: better access to distal aneurysms; minimal aneurysmal manipulation and risk of rerupture, and the possibility of treating multiple aneurysms. Furthermore, compared to surgery, EVT is less invasive as it avoids craniotomies, and may be performed under local anaesthesia, which allows functional testing of the patient before and after vessel occlusion [12]. Parent artery occlusion can be tolerated if the aneurysm is located distally, especially when there is good collateral circulation. EVT has evolved over the last few years. There are significant improvements in endovascular coils, catheters, and techniques, and thus, parent vessel occlusion is not always necessary. The decision to use a coil or cyanoacrylate as an occlusive device depends on IIA location.

The management of an un-ruptured IIA is also not clear. Currently, literature reports include several cases that have been monitored with serial angiography with resolution or decrease in size of IIAs with appropriate antibiotic therapy alone [9] Delayed surgery may reduce the risk of perioperative rupture. Unfortunately, it is not yet known with certainty which IIAs are at imminent risk of haemorrhage [11]. Given that the risk of rupture is higher during the acute phase, delayed intervention can be associated with catastrophic consequences.

\section{General discussion and clinical implications}

Prof. Agnelli, Dr. Hamam, Dr. Pasquino, Dr. Alberti: Here, we report the case of a 20 -year-old woman with acute infective endocarditis who experienced neurological complications due to the rupture of an unknown IIA after cardiac surgery. IIAs are rare but potentially fatal cerebrovascular complications of bacterial endocarditis. Septic embolisation that precedes aneurysmal development is often clinically silent; reported neurological symptoms related to the presence of the IIAs are very frequently non-specific. Given this, these aneurysms are very often suspected only after their rupture which very often produces catastrophic consequences. Moreover, heparinisation during a surgical cardiac approach or postoperative anticoagulant therapy can foster the rupture of unknown aneurysms. Physicians must rule out the possibility of IIA in all patients with bacterial endocarditis. In the absence of clinical signs or symptoms of IIAs, routine screening with neuroimaging has been thought to not usually be warranted [13]. In the case reported on here, IIA was asymptomatic. Thus, we advise carrying out neuroimaging even in the presence of asymptomatic neurological patients with leftsided bacterial endocarditis. If such patients are deemed candidates for cardiac surgery within the first 4 weeks from starting antibiotic therapy, the utility of non-invasive MRA for detecting IIAS is justified. Moreover, in the presence of a clinical case with symptoms, a cerebral angiography is recommended.

In the patient here, EVT of a ruptured large aneurysm was performed. Currently, there are no accepted guidelines regarding the management of IIAs as no randomised controlled trials have been carried out to date to determine such. Presently, although literature reports include only a very limited number of reported cases, the EVT of IIAs appears to be an effective technique due to its low complication rate. In a series of 14 patients all of the 18 IIAs were successfully occluded with endovascular therapy without neurological complications [4]. In another series involving 16 patients with ruptured IIAs, EVT was reported to have produced benefits in 14 cases [14]. In a third study, EVT was performed on six aneurysms and completed on five aneurysms. Follow-up angiography performed on the four survivors revealed no recurrences, and no re-treatments were needed. None of these latter four patients experienced bleeding from their aneurysms [15]. In our patient, endovascular embolisation of IIA was preferred over surgery given that the aneurysm was located distally in the cerebral hemispheres, and there was a high potential risk of re-rupture. Following EVT embolisation, our patient underwent craniotomy for the evacuation of 
intraparenchymal haematomas as her clinical condition had deteriorated due to the mass effect of the hemorrhagic lesion. The presence of a large intracranial haematoma could be an indication for a direct surgical approach. Few cases have reported on the choice of EVT in the presence of intracranial haematoma [15]. We believe that, when the clinical condition permits, the presence of an intracranial haematoma should not be a contraindication to performing EVT.

In conclusion, we have the following suggestions for improving clinical practice:

- As the development of IIAs is possible in all patients with bacterial endocarditis, especially those requiring cardiac surgery within the first 4 weeks of antimicrobial therapy, we suggest performing non-invasive MRA on patients without neurological manifestations, while in the presence of neurological manifestations, cerebral angiography is recommended.

- Early intervention should be considered for all IIA patients. IIAs should be treated prior to cardiac surgery as they pose a high risk of rupture, which in turn can increase with anticoagulation treatment administered during cardiac surgery.

- EVT appears to be both reliable and safe for IIAs and thus should be regarded as a first-choice technique.

Conflict of interest statement The authors declare that they have no conflict of interest related to the publication of this manuscript.

\section{References}

1. Snygg-Martin U, Gustafsson L, Rosengren L et al (2008) Cerebrovascular complications in patients with left-sided infective endocarditis are common: a prospective study using magnetic resonance imaging and neurochemical brain damage markers. Clin Infect Dis 47:23-30
2. Leipzig TJ, Brown FD (1985) Treatment of mycotic aneurysms. Surg Neurol 23:403-407

3. Clare CE, Barrow DL (1992) Infectious intracranial aneurysms. Neurosurg Clin N Am 3:551-566

4. Chapot R, Houdart E, Saint-Maurice JP et al (2002) Endovascular treatment of cerebral mycotic aneurysms. Radiology 222:12031213

5. Kannoth S, Iyer R, Sanjeev V et al (2007) Intracranial infectious aneurysm: presentation, management and outcome. J Neurol Sci 256:3-9

6. Vilacosta I, Graupner C, San Roman JA et al (2002) Risk of embolization after institution of antibiotic therapy for infective endocarditis. J Am Coll Cardiol 39:1489-1495

7. Ahmadi J, Tung H, Giannotta SL, Destian S (1993) Monitoring of infectious intracranial aneurysms by sequential computed tomographic/magnetic resonance imaging studies. Neurosurgery 32:45-49

8. Scotti G, Li MH, Righi C et al (1996) Endovascular treatment of bacterial intracranial aneurysms. Neuroradiology 38:186-189

9. Peters PJ, Harrison T, Lennox JL (2006) A dangerous dilemma: management of infectious intracranial aneurysm complicating endocarditis. Lancet Infect Dis 6:742-748

10. Asai T, Usui A, Miyachi S, Ueda Y (2002) Endovascular treatment of intracranial mycotic aneurysm prior to cardiac surgery. Eur J Cardiothorac Surg 948-950

11. Frizzell RT, Vitek JJ, Hill DL, Fisher WS (1993) Treatment of a bacterial (mycotic) intracranial aneurysm using an endovascular approach. Neurosurgery 32:852-854

12. Cloft HJ, Kallmes DF, Jensen ME, Lanzino G, Dion JE (1999) Endovascular treatment of ruptured, peripheral cerebral aneurysms: parent artery occlusion with short Guglielmi detachable coils. AJNR Am J Neuroradiol 20:308-310

13. Baddour LM, Wilson WR, Bayer AS et al (2005) Diagnosis, antimicrobial therapy, and management of complications: a statement for healthcare professionals from the Committee on Rheumatic Fever, Endocarditis, and Kawasaki Disease, Council on Cardiovascular Disease in the Young, and the Councils on Clinical Cardiology, Stroke, and Cardiovascular Surgery and Anesthesia, American Heart Association: endorsed by the Infectious Diseases Society of America. Circulation 11:394-434

14. Chun JY, Smith W, Halbach VV et al. (2001) Current multimodality management of infectious intracranial aneurysms: a series of 16 cases $48.1203-13$

15. Phuong LK, Link M, Wijdicks E (2002) Management of intracranial infectious aneurysms: a series of 16 cases. Clinical studies. Neurosurgery 51(5):1145-1152 